\title{
Enquête
}

Archives de la revue Enquête

6 | 1991

La socialisation de la jeunesse

\section{Jeunesse et habitus}

Pertinence de l'hypothèse, impertinence des enquêtes

\section{Roger Bénoliel et Roger Establet}

\section{CpenEdition}

Journals

Édition électronique

URL : http://journals.openedition.org/enquete/120

DOI : 10.4000/enquete. 120

ISSN : 1953-809X

Éditeur :

Cercom, Éditions Parenthèses

Édition imprimée

Date de publication : 2 juin 1991

Référence électronique

Roger Bénoliel et Roger Establet, « Jeunesse et habitus », Enquête [En ligne], 6 | 1991, mis en ligne le 08 février 2006, consulté le 10 décembre 2020. URL : http://journals.openedition.org/enquete/120 ; DOI : https://doi.org/10.4000/enquete. 120

Ce document a été généré automatiquement le 10 décembre 2020. 


\title{
Jeunesse et habitus
}

\author{
Pertinence de l'hypothèse, impertinence des enquêtes
}

\author{
Roger Bénoliel et Roger Establet
}

Le rituel qui présida en 1978 au choix de notre thème d'enquête fut une forme d'expression démocratique post-soixante-huitarde: il était alors hors de question d'imposer magistralement le sujet. La grande grève de 1976 avait ranimé les souvenirs, et l'autorité pédagogique ne pouvait s'affirmer que par la ruse et au terme de laborieuses négociations. Le sujet du questionnaire fut adopté, au terme de trois heures de discussions, par 12 voix sur 70. Devant ce résultat, qui imposait à tous un sujet choisi par une minorité, sans qu'un accord minimal ait pu se faire sur une solution de compromis, nous décidâmes l'année suivante de revenir à l'autorité pédagogique ouverte, en proposant un sujet relativement général que le premier trimestre permettait de préciser sur la base de la réflexion des étudiants. Ce choix de 12 sur 70 - et on écrirait presque contre 52, puisque les minoritaires ont tenu jusqu'au bout à leur idée - illustre le concept de démocratie tel que les assemblées de 1968 l'avaient popularisé : il s'agissait avant tout de la libre expression, accomplie comme valeur en soi, dans une certaine indifférence pour les formules finales de décision collective. En brontosaures de la démocratie de la volonté générale, nous étions décontenancés par une telle insouciance. Nous avons rétabli l'ordre pédagogique, et, nous en fûmes surpris, sans la moindre difficulté. Aujourd'hui que sont toutes disciplines restituées, le rituel qui a organisé notre première séance de TP, de 1973 à 1976, paraîtrait incongru. Nos étudiants de licence, qui ont fait l'enquête l'année précédente, viennent nous trouver, en nous demandant quel sujet nous avons choisi cette année, et les nouveaux attendent comme une surprise que nous leur proposions notre choix magistral. On mesure par là à quel point le système scolaire et familial a su rétablir l'ordre en quelques années. À bien y réfléchir, c'est un ordre différent. Le rapport pédagogique, décrit par P. Bourdieu et J.-C. Passeron dans Les Héritiers, fondé sur la mutuelle reconnaissance des charismes peut difficilement se donner carrière dans des unités collectives qui ont pris la taille de petites villes (20 000 étudiants à la faculté des lettres d'Aix-en-Provence), où les rituels bureaucratiques des inscriptions sont popularisés par la télévision et où la démographie a imposé bon nombre de rationalisations. Surtout, les lycées ont préparé les étudiants à accepter l'autorité 
enseignante, sous la condition que le jeune voie ses droits de personne privée reconnus : la forme de politesse préférée des lycéens est l'interpellation de l'élève par «Prénom, tu...» à laquelle répond dissymétriquement «Monsieur, vous...» Arrivés à la fac, les jeunes accèdent pour la première fois à l'interpellation par le vous, mais avec une longue pratique de la dissymétrie. L'autorité n'a plus que faire de la ruse, le sourire suffit. On espère que ces remarques informelles, pour introduire la rhapsodie de nos enquêtes, ne passeront pas pour de l'impressionnisme hors sujet: les formes d'interpellation entrent dans la définition sociale du rapport pédagogique et du statut de la jeunesse, qu'il s'agisse du symétrique et solennel «Citoyen, tu...» attesté à Normale supérieure sous la Convention nationale, au " Vous/Vous » distancé, laïque et obligatoire de notre jeunesse, jusqu'à cet étrange « Tu/Vous » accepté de bonne grâce par nos enfants. Surtout, entre 1976 et aujourd'hui, quatorze enquêtes différentes nous ont donné l'occasion de réfléchir en sociologues aux modes d'apprentissage et de socialisation de quelque 1500 étudiants.

Jeunesse et affectivité : les leçons d'une enquête ratée

2 L'enquête de 1978 portait sur la gestion sociale de l'affectivité chez les jeunes: leurs attitudes et opinions vis-à-vis des formes alternatives de vie en couple, leurs acceptations ou rejets des formes marginales ou déviantes de vie privée, le concubinage... Dès la réception des questionnaires, il apparaissait que l'enquête, bien que conduite dans les règles de l'art, était ratée. On pouvait se fier à deux critères empiriques: les questions ouvertes ne donnaient lieu qu'à un petit nombre de réponses, toujours les mêmes; les questions fermées agglutinaient toute la population autour des mêmes items. Les résultats donnaient une impression d'uniformité et de grisaille: non, on ne parlait pas souvent de sujets de ce genre; on ne s'intéressait pas à ce genre de problèmes; on se souciait bien davantage des études, de la profession que l'on occuperait demain ; oui, bien sûr, on tolérait ce que l'on voudra, couples homosexuels ou concubinage ; mais, quant à nous, non, vraiment, on ne s'occupe pas de ces problèmes-là. Peu d'écarts entre les filles et les garçons; pas de différence entre les jeunes lycéens et les jeunes travailleurs: le désespoir pour le sociologue.

3 À cette indifférence, deux explications possibles : ou bien, le thème ne concernait pas la population interrogée; ou bien, au contraire, il s'agissait d'une réticence volontaire destinée à éloigner l'interrogateur indiscret. Tous les indices objectifs le montrent : c'est précisément cette génération de jeunes qui a rompu avec les modèles familiaux traditionnels de gestion familiale des affects et de la sexualité. La seconde hypothèse est donc meilleure. Et, à bien relire tous ces tableaux ratés, il y a en trois qui, rapprochés en un seul, la confirment.

Tableau 1. Ont discuté de problèmes touchant aux affects et à la sexualité

\begin{tabular}{|lccc|}
\hline (en $x$ ) & en famille & avec des copains & avec un $(\theta)$ ami $(\theta)$ \\
lycéens & 37 & 39 & 60 \\
lycéennes & 49 & 58 & 79 \\
actils & 31 & 54 & 67 \\
actives & 55 & 56 & 73 \\
\hline
\end{tabular}

4 Les jeunes se décrivent selon les normes nouvelles de la tolérance la plus complète : reste que les affects et la sexualité ne sont pas pour autant passés dans le domaine public, demeurent dans la sphère du confidentiel et, conformément aussi à la tradition, plus nettement pour les garçons que pour les filles. Les révolutions morales, comme les autres, 
ne brisent pas simultanément toutes les structures du passé. Notre enquête mesurait mal les changements, sinon pour en montrer le caractère « mesuré ».

L'occupation juvénile de l'espace festif

5 Le travail de l'année 1985 a pour origine l'inspiration que suscita, chez une de nos étudiantes de maîtrise, Fatiah Amara, une courte et profonde réflexion de Michel Verret, intitulée "Ce qu'un sociologue pourrait bien dire de la fête». Il était passionnant de réétudier les clivages instaurés par la scolarité, l'origine sociale et le sexe dans une classe d'âge, en prenant pour fil directeur ce mixte social de spontanéité et d'organisation, de jeu de rôle et de jeu sur les rôles, de plaisirs et d'obligations, que constitue le temps fort de la fête. Dans une série d'entretiens, menés en 1984, Fatiah Amara permettait de formuler ainsi quelques hypothèses de variation. Côté lycéen d'enseignement général, côté cadre supérieur et féminin, la fête exprime une sociabilité intimiste, avec reconnaissance personnelle des partenaires. Côté lycée professionnel, origine ouvrière et masculin, la fête introduit à un univers plus « fusionnel », où la reconnaissance mutuelle n'implique au même degré l'interconnaissance personnelle. Opposition dans le style même de fête valorisée, et dans les ingrédients festifs mis en œuvre. L'enquête statistique nuance le constat qualitatif, en mettant en évidence bon nombre de points d'accord, parfaitement positifs entre les jeunes de tous les milieux et de tous les établissements. Sur les fêtes préférées, qui excluent les adultes et les institutions. Les copains, d'abord.

Tableau 2. L'ordre des réjouissances

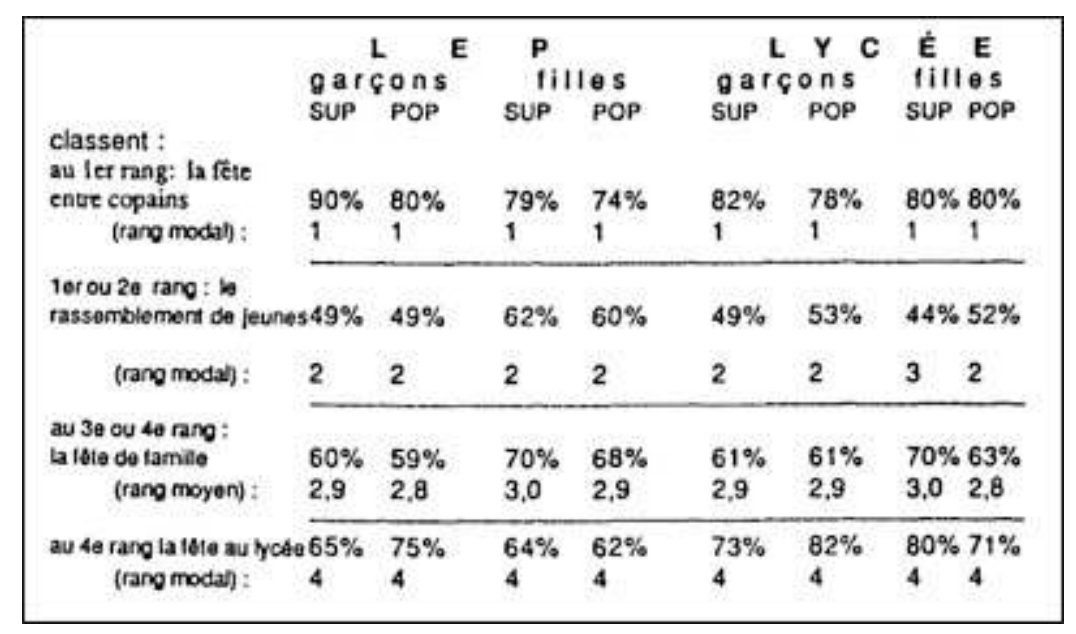

6 Le lycée est un lieu d'étude, non point un lieu de vie, qu'il faudrait investir ou subvertir par la fête. Et la famille est devenue un territoire d'affectivité tranquille, où on laisse le soin aux adultes d'organiser des festivités qui laissent des impressions mitigées.

Tableau 3. Le charme modéré des fêtes de famille

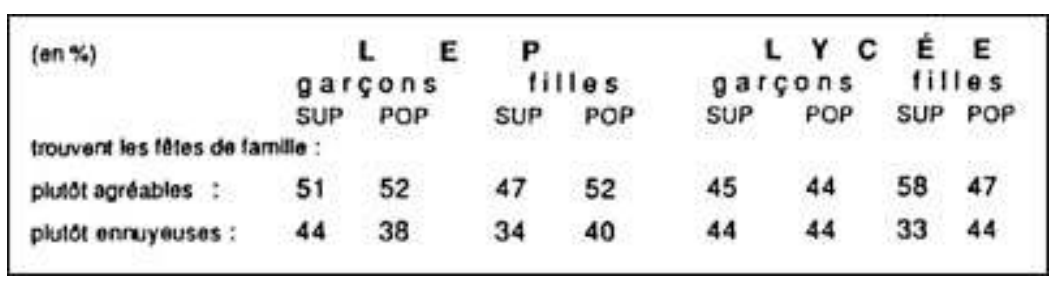

7 La fête entre copains n'est pas pour autant une transgression clandestine : dans tous les milieux, les participants sont connus des parents; les interdictions parentales sont 
exceptionnelles ; le droit festif des jeunes est donc un droit reconnu, qui donne rarement lieu à des récriminations de la part des adultes ou des parents.

Tableau 4. Le regard éloigné et bienveillant de la famille

\begin{tabular}{|c|c|c|c|c|c|c|c|c|}
\hline \multirow[t]{3}{*}{$(e n \%)$} & $\mathbf{L}$ & $\mathbf{E}$ & \multicolumn{2}{|l|}{$\mathbf{P}$} & I & $\mathbf{Y} \mathrm{C}$ & $\mathbf{E}$ & $\mathbf{E}$ \\
\hline & \multicolumn{2}{|c|}{ garçons } & \multicolumn{2}{|c|}{ filles } & \multicolumn{2}{|c|}{ gargons } & \multicolumn{2}{|c|}{ filles } \\
\hline & SUP & POP & Sup & POP & sup & POP & SuP & POP \\
\hline $\begin{array}{l}\text { tes parems connaissent } \\
\text { les inveds de la iste : }\end{array}$ & 72 & 68 & 84 & 70 & 62 & 56 & 75 & 64 \\
\hline $\begin{array}{l}\text { ont invite (ont ote irvitt0s) } \\
\text { au domicile des parents : }\end{array}$ & $\begin{array}{l}\text { pour ur } \\
88\end{array}$ & $\begin{array}{l}\text { no teie } \\
79\end{array}$ & 83 & 78 & 94 & 87 & 99 & 90 \\
\hline $\begin{array}{l}\text { - les parents ont interda } \\
\text { de participer a une tele : }\end{array}$ & 19 & 13 & 25 & 36 & 23 & 18 & 29 & 24 \\
\hline $\begin{array}{l}\text { ont particips a une lete quil } \\
\text { protestations of adultes : }\end{array}$ & $\begin{array}{l}42 \\
42\end{array}$ & $\begin{array}{l}\text { nd lieu a d } \\
35\end{array}$ & 30 & 25 & 35 & 31 & 27 & 25 \\
\hline (dt pectestations des parents) & 21 & 11 & 17 & 11 & 18 & 16 & 16 & 12 \\
\hline
\end{tabular}

8 La fête s'accomplit dans un espace libéré par les adultes, loin des contraintes familiales ou scolaires. Elle permet la mise en jeu de modèles et de normes qui ont valeur générale.

Tableau 5. Le meilleur souvenir de fête en 1985 (question ouverte)

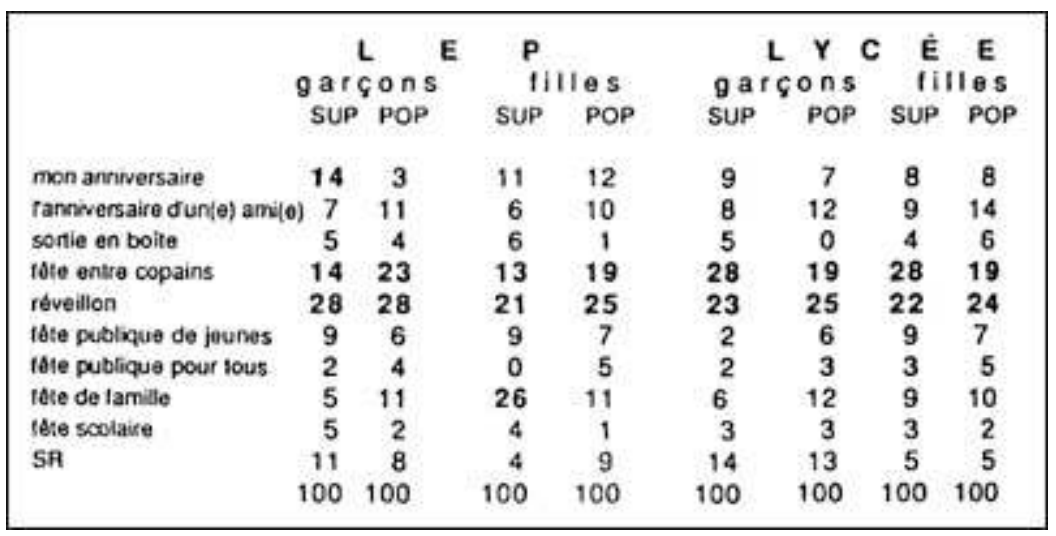

9 La question ouverte, volontairement narrative, laisse place aux aléas de l'année écoulée. Elle ne met que mieux en évidence le système commun de préférences: moment privilégié, identité de génération. La fête est chose sérieuse: une simple «sortie en boîte » ne suffit pas à la fixer dans les mémoires. Le réveillon est le meilleur modèle. On se prépare à une fête: on lance les invitations quelques jours à l'avance, on cherche à connaître le nom des participants, on revêt des habits particuliers, on consacre du temps à sa toilette. 
Tableau 6. Les préparatifs

\begin{tabular}{|c|c|c|c|c|c|c|c|c|}
\hline \multirow{3}{*}{ (en $\%$ ) } & $t$ & E & \multicolumn{2}{|l|}{$\mathbf{p}$} & & Y C & $\dot{\mathrm{E}}$ & $\mathbf{E}$ \\
\hline & \multicolumn{2}{|c|}{ garçons } & \multicolumn{2}{|c|}{ filles } & \multicolumn{2}{|c|}{ garçons } & \multicolumn{2}{|c|}{ filles } \\
\hline & sup & POP & Sup & POP & sup & POP & Sup & POP \\
\hline \multicolumn{9}{|l|}{ Invitation lancele : } \\
\hline le jour męrne & 7 & 8 & 6 & 2 & 6 & 7 & 3 & 2 \\
\hline $\begin{array}{l}\text { une semaine (ou +) avant } \\
\text { cherchent a savoir la nom }\end{array}$ & 47 & 55 & 57 & 67 & 58 & 52 & 59 & 70 \\
\hline $\begin{array}{l}\text { des participants } \\
\text { mettert des vetements }\end{array}$ & 48 & 52 & 59 & 55 & 41 & 45 & 55 & 62 \\
\hline $\begin{array}{l}\text { particuliers } \\
\text { consacrent a se préparer: }\end{array}$ & 53 & 58 & 63 & 63 & 46 & 42 & 65 & 61 \\
\hline - uno demi heuto ou plus & 60 & 73 & 96 & 84 & 62 & 65 & 86 & 88 \\
\hline - une heure ou plus & 23 & 14 & 66 & 50 & 17 & 18 & 45 & 49 \\
\hline
\end{tabular}

10 La réussite d'une fête dépend de quelques éléments sur lesquels, à peu de choses près, tous les jeunes s'accordent.

Tableau 7. Les trois composantes essentielles d'une fête

\begin{tabular}{|c|c|c|c|c|c|c|c|c|}
\hline \multirow[t]{3}{*}{$(e n \%)$} & \multicolumn{2}{|c|}{ L $E$} & \multicolumn{2}{|l|}{$\mathbf{P}$} & \multirow{2}{*}{\multicolumn{2}{|c|}{$\underset{\text { garçons }}{\mathrm{Y}} \mathrm{C}$}} & \multirow{2}{*}{\multicolumn{2}{|c|}{$\underset{f(i f l e s}{E} \underset{0}{E}$}} \\
\hline & \multicolumn{2}{|c|}{ gargons } & \multicolumn{2}{|c|}{ filles } & & & & \\
\hline & SUP & POP & SuP & POP & sup & & SUP & POP \\
\hline $\begin{array}{l}\text { La musique. } \\
\text { au premier rang }\end{array}$ & 51 & 55 & 49 & 55 & 49 & 48 & 58 & 64 \\
\hline $\begin{array}{l}\text { au 1er ou } 2 \text { ème rang } \\
\text { Ladanso. }\end{array}$ & 79 & 74 & 70 & 76 & 73 & 70 & 80 & 95 \\
\hline $\begin{array}{l}\text { A fun des tois premiats rangs } \\
\text { La discussion. }\end{array}$ & 1549 & 62 & 70 & 72 & 53 & 55 & 71 & 72 \\
\hline A fun des trois premiers rangs & 30 & 38 & 34 & 34 & 38 & 36 & 45 & 52 \\
\hline
\end{tabular}

11 Et ils ne s'accordent pas moins sur les éléments qu'ils jugent accessoires (les jeux, les farces, les déguisements) ou nuisibles (l'alcool, avec une défiance plus marquée chez les filles). Bref, une fête est une sorte de célébration de la sociabilité de génération. Et si l'on n'apprécie guère la fête à l'école, ce sont les camarades de classe que l'on rencontre d'abord dans une fête.

Tableau 8. La sociabilité festive

\begin{tabular}{|c|c|c|c|c|c|c|c|c|}
\hline \multirow[t]{3}{*}{$(\theta n \%)$} & \multirow{2}{*}{\multicolumn{2}{|c|}{ gatçons }} & $\mathbf{p}$ & & \multirow{2}{*}{\multicolumn{2}{|c|}{ garçons }} & $\dot{E}$ & E \\
\hline & & & \multicolumn{2}{|c|}{ filles } & & & \multicolumn{2}{|c|}{ filles } \\
\hline & sup & POP & sup & POP & sup & POP & Sup & POP \\
\hline \multicolumn{9}{|c|}{ ont retrouvd, bors do la dernière tate : } \\
\hline des camarades do Lycée & 56 & 62 & 57 & 49 & 74 & 66 & 71 & 64 \\
\hline (et eux seulement) & 35 & 37 & 38 & 33 & 55 & 44 & 52 & 50 \\
\hline des voising & 37 & 43 & 36 & 34 & 27 & 35 & 26 & 21 \\
\hline ont rencontré des inconnu. & j59 & 82 & 74 & 87 & 88 & 85 & 90 & 84 \\
\hline
\end{tabular}

12 On remarquera, sans surprise, la sociabilité moins restreinte à l'école des élèves des LEP. Les carrières, dans un LEP, sont plus brèves et plus précaires surtout que dans un lycée général. Mais le tableau révèle plus de normes que de variations: dans la fête, les sociabilités juvéniles s'affirment et, éventuellement, s'étendent, sur la base d'un noyau solide d'interconnaissance préalable.

13 Dans ce camaïeu de roses, où « festif » ne rime point avec « orgiaque » ou « transgressif », comme dans les surboums des films de la "nouvelle vague», s'inscrivent quelques variations. On en aura noté quelques-unes à propos des fêtes préférées, du temps de 
préparation. Les qualités attribuées à une tenue de fête permettent de distinguer sur un premier axe l'originalité, plus prisée des lycéens de milieu favorisé à l'esthétique, soulignée par tous les élèves de LEP; et sur un second, opposant le conformisme vestimentaire des déclassés (garçons fils de cadres scolarisés en LEP) à la simplicité revendiquée par les intégrées (filles de cadres scolarisées en lycée). Dans l'ensemble, les élèves de LEP s'appliquent davantage à marquer la rupture entre le temps festif et le temps ordinaire, et les lycéens soulignent les continuités. Plus la semaine de la vie est sérieuse, plus on demande au dimanche d'être endimanché.

Tableau 9. Les qualités attribuées à une tenue de fête

\begin{tabular}{|c|c|c|c|c|c|c|c|c|}
\hline \multirow[t]{3}{*}{ (en \%) } & t & $\mathbf{E}$ & \multicolumn{2}{|l|}{$\mathbf{P}$} & & $\mathbf{Y} \mathrm{C}$ & $\dot{\mathbf{E}}$ & $\mathbf{E}$ \\
\hline & \multicolumn{2}{|c|}{ garçons } & \multicolumn{2}{|c|}{ filles } & \multicolumn{2}{|c|}{ garcons } & \multicolumn{2}{|c|}{ lilles } \\
\hline & SUP & POP & SuP & POP & SUP & POP & SUP & POP \\
\hline esthatique & 28 & 33 & 37 & 32 & 24 & 24 & 16 & 25 \\
\hline fantaisie & 14 & 5 & 10 & 9 & 11 & 9 & 9 & 9 \\
\hline décontraction & 8 & 22 & 18 & 23 & 20 & 25 & 21 & 27 \\
\hline simplicte & 22 & 24 & 16 & 20 & 18 & 18 & 24 & 12 \\
\hline originaline & 8 & 6 & 8 & 7 & 13 & 8 & 20 & 11 \\
\hline conlormisme & 20 & 10 & 11 & 9 & 14 & 16 & 10 & 16 \\
\hline
\end{tabular}

Mais il s'agit là de nuances, et non de clivages. C'est l'impression générale qui résulte de tous les indicateurs de l'enquête (occasions de célébration, éléments préférés, préparatifs, nombre et qualité des participants, relations avec les adultes). Une seule exception: le lieu idéal pour organiser une fête avec des amis.

Tableau 10. Le lieu idéal pour organiser une fête entre amis

\begin{tabular}{|c|c|c|c|c|c|c|c|c|}
\hline \multirow[t]{2}{*}{$(e n \%)$} & \multirow{2}{*}{\multicolumn{2}{|c|}{$\begin{array}{l}\text { garçons } \\
\text { SUP POP }\end{array}$}} & \multicolumn{2}{|c|}{ filles } & \multicolumn{2}{|c|}{$\underset{\text { garçons }}{\mathbf{L}} \mathbf{C}$} & \multicolumn{2}{|c|}{$\begin{array}{l}E \text { E } \\
\text { filles }\end{array}$} \\
\hline & & & SUP & POP & SuP & POP & SUP & POP \\
\hline $\begin{array}{l}\text { chez sol } \\
\text { chez des amis }\end{array}$ & $\begin{array}{l}9 \\
2\end{array}$ & $\begin{array}{l}3 \\
6\end{array}$ & $\begin{array}{r}13 \\
6\end{array}$ & $\begin{array}{l}6 \\
6\end{array}$ & $\begin{array}{l}16 \\
14\end{array}$ & $\begin{array}{r}12 \\
3\end{array}$ & $\begin{array}{l}18 \\
14\end{array}$ & $\begin{array}{l}16 \\
10\end{array}$ \\
\hline en botte & 5 & 11 & 9 & 6 & 4 & 2 & 3 & 3 \\
\hline un grand beal & 37 & 45 & 43 & 46 & 21 & 31 & 23 & 27 \\
\hline un peth local & 19 & 16 & 11 & 14 & 11 & 15 & 15 & 8 \\
\hline en plein air & 5 & 6 & 4 & 8 & 6 & 10 & 4 & 8 \\
\hline maison, villa & 14 & 5 & 8 & B & 16 & 18 & 18 & 16 \\
\hline Sans réponse & 9 & 8 & 6 & 6 & 12 & 9 & 5 & 12 \\
\hline
\end{tabular}

15 Ici, on a bien affaire à des clivages : le lieu idéal de la fête est défini comme un lieu public dans $61 \%$ des cas par les élèves les plus typiques des LEP (les garçons d'origine populaire) et par des majorités importantes de leurs condisciples, alors qu'il est défini comme un lieu privé (maison, villa, chez moi, chez des amis) par les lycéens enfants de cadre (46\% des garçons et $50 \%$ des filles, soit respectivement $52 \%$ et $55 \%$ des répondants). Et, comme il est prévisible dans un conflit de normes, les lycéens et lycéennes d'origine populaire occupent une position intermédiaire. On peut lire dans ce tableau, à l'aide de l'analyse factorielle, une opposition secondaire entre les amateurs de l'intérieur et les partisans du plein air, où se comptent plus nombreux que les autres les atypiques des lycées (enfants de milieu populaire, surtout les garçons) et des LEP (les fils de cadres).

16 En ce point du compte rendu d'enquête, le sociologue intéressé à maximiser les différences pourrait révoquer en doute les impressions continuistes données par les 
tableaux précédents. Après tout, les éléments prennent leurs sens du contexte où ils sont introduits. Et ne tenons-nous pas là deux types d'organisation juvéniles opposés: les lycéens retrouvent le modèle de la réception; les élèves des LEP mettent en place une fête populaire autogérée. Rien n'interdirait de lire, malgré des analogies de surface, l'action de valeurs contrastées et de déceler la formation d'habitus de classe. De fait, une analyse factorielle des correspondances, portant sur l'ensemble des indicateurs, marque bien une opposition fondamentale entre les garçons de milieu populaire scolarisés en LEP et les lycéens et les lycéennes de milieu bourgeois, avec un axe secondaire analogue à celui qui se lit dans notre tableau (les intégrés d'un côté, les déclassés et les promus de l'autre). Côté LEP, valorisation du lieu public, du réveillon, des qualités esthétiques de la tenue de fête, la présence des voisins ; côté lycéen, le lieu privatif, la tenue décontractée et/ou originale, la participation plus exclusive de camarades de classe. Côté promus et déclassés, une plus forte insistance sur le conformisme vestimentaire, le plein air et la célébration des anniversaires.

Graphique 1. Analyse factorielle de correspondances

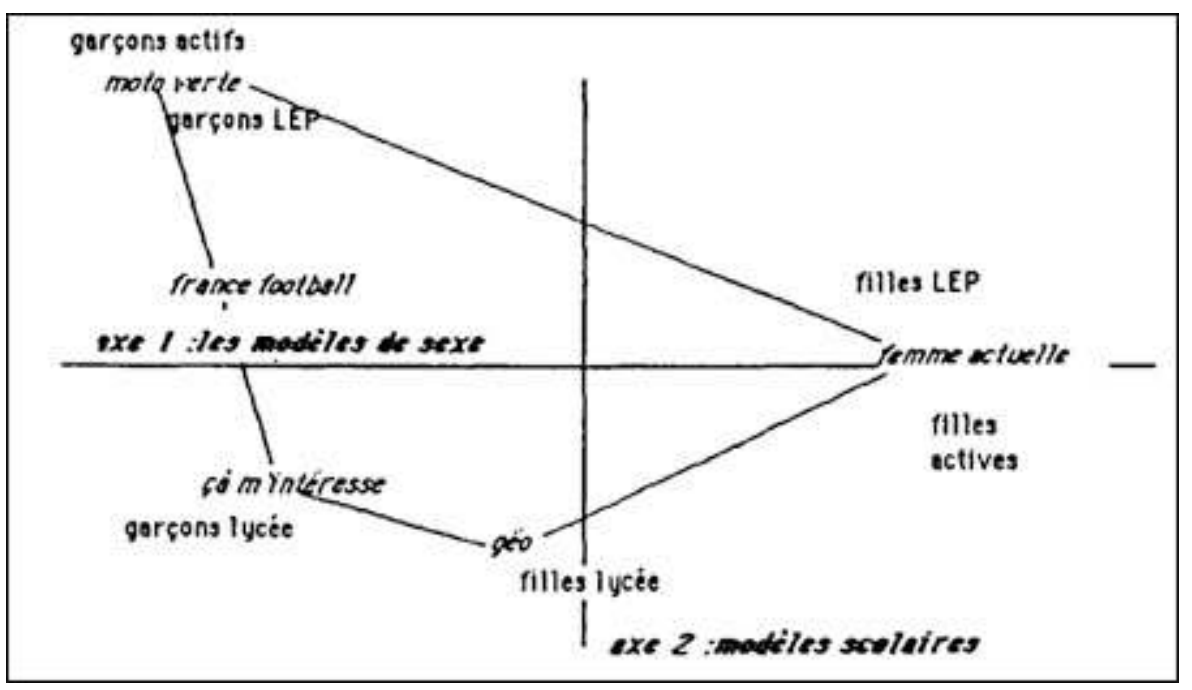

Il serait bien imprudent de conclure, croyons-nous, à l'existence de deux habitus de classe, fussent-ils en germe, sur la foi de ces tendances statistiques. Au lycée comme au LEP, les individus qui cumulent tous les traits de l'un ou l'autre type idéal sont seulement quelques unités. C'est à propos de l'institution totalitaire des " convicts » monastiques que Durkheim (1969) introduisait le concept d'habitus, fruit d'un travail assidu de la conversion scolaire. Nos sociétés modernes ne connaissent qu'exceptionnellement de telles institutions. Même si dans la formation de la personne à la relation sociale, l'école et la famille imposent les contraintes et les frontières les plus fortes, elles ne sont pas sans concurrence (modèles médiatiques, société des pairs de génération). Elles ne sont pas davantage totalement cohérentes entre elles. L'espace libéré pour la fête n'est pas immédiatement rempli par des normes qui différencieraient et sépareraient les milieux, en assujettissant implacablement les individus.

Musique, lecture et cinéma : habitus buissonnant et habitus buissonnier

Ne soyons pas ingrats. Le concept d'habitus nous a fourni plus d'une hypothèse, dans la construction de nos enquêtes sur les jeunes. L'idée que les apprentissages puissent se transférer, au-delà du domaine où ils ont été directement inculqués et contrôlés (Bourdieu \& Passeron, 1970), est une idée féconde propre à rendre attentifs aux effets les 
moins visibles de l'éducation, et singulièrement de l'éducation scolaire. C'est sous cette inspiration bourdivine que nous avons conçu les hypothèses de nos enquêtes sur la musique (1983), la lecture (1987) et le cinéma (1989). Et, dans les trois cas, nous avons pu repérer des transferts significatifs d'apprentissage.

La musique est l'univers culturel le plus valorisé par les jeunes de tous les milieux, celui où ils investissent le plus de sociabilité, sous forme d'innombrables prêts, emprunts, discussions, conseils, invitations et sorties. Et la force de l'inculcation scolaire se marque aussi bien dans le rapport à la pratique musicale, beaucoup plus scolaire (professeurs, partitions, exercices) chez les lycéens, que dans les préférences. Il ne s'agit pas ici d'influence directe : la musique classique n'a pas grande présence. Les élèves de LEP ont une prédilection pour l'actualité musicale et les chanteurs et les groupes les plus récents, les lycéens s'intéressent à des musiciens et des musiques plus anciennement reconnus, et parfois assez anciennement reconnus pour figurer dans les anthologies scolaires (c'était le cas pour Brel et Brassens, en tête du hit-parade lycéen). L'impression d'une orientation des goûts musicaux par des systèmes de valeurs plus généraux est renforcée par le constat, dans notre enquête, d'un effet de dominance, attesté aussi bien chez les déclassés que chez les promus: les enfants de cadres scolarisés dans un LEP modernisent leurs préférences et associent plus volontiers la musique et la danse ; à l'opposé, les enfants d'ouvriers scolarisés dans un lycée troquent Halliday et Police pour Brassens. Origine sociale et avenir objectif de classe impriment une marque profonde dans l'univers musical, si important pour les lycéens.

Avec la lecture, la recherche des habitus est peut-être trop facile : l'activité est tellement prônée par l'école, et dès le plus jeune âge, qu'on a du mal à distinguer ici les obligations et les préférences. Une chose est sûre en tout cas : les contraintes de l'examen (le bac dit " de français ») et/ou le talent persuasif de nos collègues littéraires amènent les lycéens à consacrer beaucoup de temps à la lecture, et notamment à la lecture consacrée par les anthologies scolaires, qu'il s'agisse de textes anciens ou de littérature contemporaine. À l'opposé, les élèves des LEP citent beaucoup plus souvent des œuvres popularisées par la télévision ou le cinéma. L'école impose mieux ses choix lorsqu'ils peuvent être scolairement rentables. Mais l'action de l'école va bien au-delà, si l'on en juge par les types de périodiques auxquels les enquêtés se sont dits personnellement intéressés. Cependant, les effets de la scolarisation concernent ici les modèles de comportement liés au sexe : les lycéens abandonnent un peu l'intérêt exclusif pour la presse sportive, en affinité avec les préférences masculines pour la littérature dramatique (romans policiers, espionnage, science-fiction sont les genres dominants, à $44 \%$ des livres cités par les lycéens, à $61 \%$ chez les élèves de LEP, à $48 \%$ chez les actifs). Chez les filles, la formation secondaire se traduit par une récession marquée de la presse dite "féminine ». Pour les deux sexes, cette affirmation moins tranchée des intérêts traditionnellement liés au sexe se fait au profit des publications documentaires et des magazines d'information générale. Le lycée infléchit donc les habitudes de lecture dans le sens d'une mixité, comme en témoigne le trapèze que dessine l'analyse factorielle des correspondances (cf. supra). 
Tableau 11. Genre des périodiques lus régulièrement et avec intérêt

\begin{tabular}{|c|c|c|c|c|c|c|}
\hline \multirow[t]{2}{*}{$(e n \%)$} & \multicolumn{2}{|c|}{ LYCEE } & \multicolumn{2}{|c|}{ LEP } & \multicolumn{2}{|c|}{ ACTIFS } \\
\hline & & $\mathrm{F}$ & G & $F$ & G & F \\
\hline sport & 32,0 & 7,5 & 28,7 & 1,7 & 37,2 & 4,6 \\
\hline intormation & 11,0 & 10,6 & 2,8 & 2,3 & 2,6 & 1.1 \\
\hline cinéma téle & 4,1 & 5,6 & 4,6 & 8.1 & 5,1 & 2,3 \\
\hline musique & 3.5 & 2,5 & 4.6 & 17,3 & 7,7 & 10,3 \\
\hline Iéminin & 2,3 & 18,1 & 1,9 & 30,6 & - & 58,6 \\
\hline Iechnique & 2,3 & 1,3 & 5,6 & 2.3 & 2,6 & 1,1 \\
\hline coevur & - & - & - & 2,3 & - & 1,1 \\
\hline photo & 4,7 & 1,3 & 0,9 & 0,6 & 3,8 & - \\
\hline documentairo & 21,5 & 19,4 & 5,6 & 5,2 & 3,8 & 2,3 \\
\hline bricolage & - & - & - & 1,2 & - & - \\
\hline autre & - & 4,4 & 4,6 & 1.7 & 7.7 & 2,3 \\
\hline aucun & 18,6 & 29.4 & 40,7 & 26,6 & 29,5 & 16,1 \\
\hline & 100 & 100 & 100 & 100 & 100 & 100 \\
\hline Eflectit interroge: & 156 & 150 & 148 & 149 & 70 & 74 \\
\hline
\end{tabular}

21 Musique et lecture témoignent du rôle de la scolarisation sur la genèse des habitudes culturelles, selon deux cas deux figures bien différents. Notre enquête la plus récente (1989), qui concerne le cinéma, nous en fournit encore un troisième. Voici, tout d'abord, organisés selon les dimensions principales de l'enquête, l'ensemble de nos résultats.

Tableau 12. Fréquence du visionnement de films (15 jours précédant l'enquête)

\begin{tabular}{|c|c|c|c|c|c|c|c|c|}
\hline & \multicolumn{4}{|c|}{ Lycte Professionnel } & \multicolumn{4}{|c|}{ Lycte gendral } \\
\hline & \multicolumn{2}{|c|}{ Gurçons } & \multicolumn{2}{|c|}{ Filles } & \multicolumn{2}{|c|}{ Garçons } & \multicolumn{2}{|c|}{ Filles } \\
\hline & ouvriers & autres & ouvriers & tutres & cadres & sutres & cadres & avers \\
\hline $\begin{array}{l}\text { nb de personnes interrogtes } \\
\text { Question 1: }\end{array}$ & 98 & 190 & 112 & 180 & 93 & 102 & 107 & 94 \\
\hline nb moyen de films vus au cintma & 1 & 0,7 & 1,1 & 1,2 & 1,4 & 1 & 1.1 & 1,1 \\
\hline en cessecte vidbo & 2 & 2,8 & 1,9 & 1.7 & 1.5 & 1.9 & 1,3 & 1.5 \\
\hline I la television & 6.7 & 7,8 & 7.5 & 7.2 & 6.1 & 6,8 & 5,4 & 6.1 \\
\hline r'ont vu aucen film as cintma & 48 & 52 & 44 & 41 & 34 & 44 & 44 & 34 \\
\hline en catsette vidto & 46 & 33 & so & 52 & 48 & 51 & 52 & 54 \\
\hline a la television & 8 & 5 & 12 & 9 & 12 & 8 & 14 & 9 \\
\hline ont vu plus de 2 films au cinkms & 28 & 28 & 27 & 31 & 39 & 30 & 33 & 29 \\
\hline ont vu plus de 2 films a is TV & 32 & 43 & 41 & 40 & 24 & 32 & 22 & 30 \\
\hline
\end{tabular}

Tableau 13. Intérêt pour le cinéma reconnu

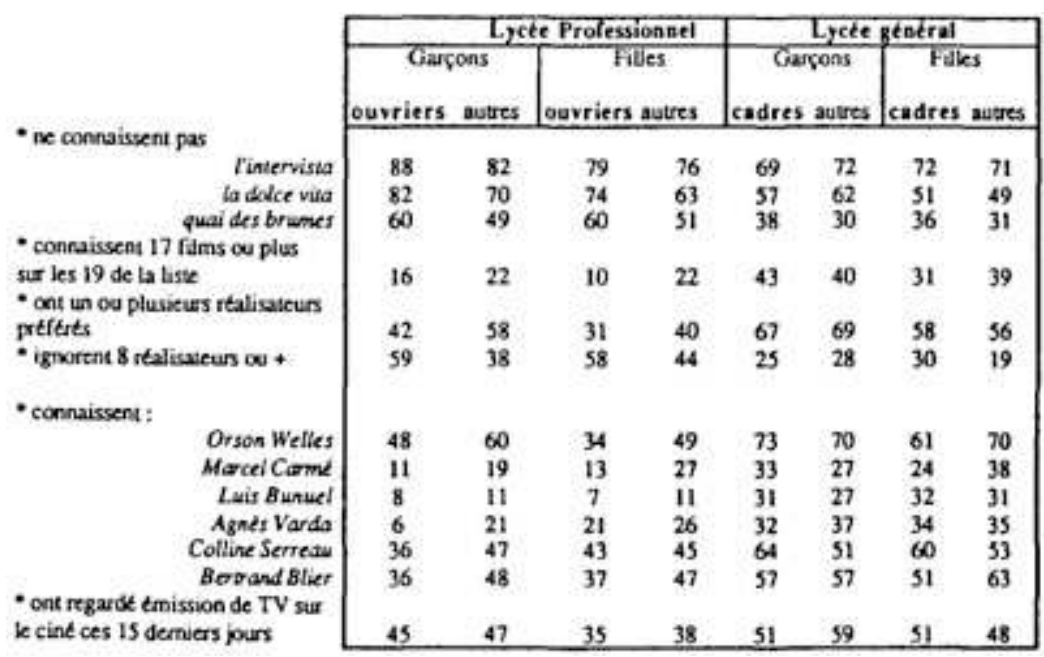


Tableau 14. Application de schèmes scolaires au cinéma

\begin{tabular}{|c|c|c|c|c|c|c|c|c|}
\hline \multirow{5}{*}{ 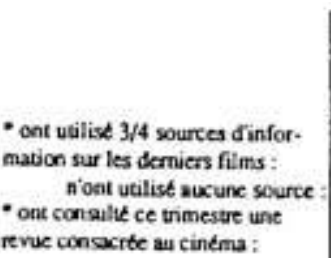 } & \multicolumn{4}{|c|}{ Lycte Prolessionatel } & \multicolumn{4}{|c|}{ Lycte gtotral } \\
\hline & \multicolumn{2}{|c|}{\begin{tabular}{|c|} 
Gargons \\
ouvriers autres \\
\end{tabular}} & \multicolumn{2}{|c|}{$\begin{array}{c}\text { Filles } \\
\text { ouvriersoutres }\end{array}$} & \multicolumn{2}{|c|}{$\begin{array}{c}\text { Gançons } \\
\text { cadres autres }\end{array}$} & \multicolumn{2}{|c|}{$\begin{array}{c}\text { Falles } \\
\text { cadres avtes }\end{array}$} \\
\hline & 10 & 21 & 21 & 28 & 29 & 21 & 26 & 37 \\
\hline & 17 & 13 & 11 & 12 & 15 & 16 & 10 & 12 \\
\hline & 32 & 46 & 33 & 44 & 53 & 45 & 57 & 52 \\
\hline $\begin{array}{r}\text { preterent la vo } \\
\text { prefierest ke doublage }\end{array}$ & 85 & $\begin{array}{l}16 \\
70\end{array}$ & $\begin{array}{l}12 \\
78\end{array}$ & ${ }_{72}^{15}$ & $\begin{array}{l}40 \\
46\end{array}$ & $\begin{array}{l}29 \\
51\end{array}$ & $\begin{array}{l}48 \\
41\end{array}$ & $\begin{array}{l}31 \\
55\end{array}$ \\
\hline $\begin{array}{l}\text { plubot distrayant } \\
\text { pluwbs serieux }\end{array}$ & $\begin{array}{l}46 \\
38\end{array}$ & $\begin{array}{l}43 \\
40\end{array}$ & $\begin{array}{l}44 \\
45\end{array}$ & $\begin{array}{l}37 \\
52\end{array}$ & $\begin{array}{l}21 \\
56\end{array}$ & $\begin{array}{l}26 \\
51\end{array}$ & $\begin{array}{l}28 \\
55\end{array}$ & $\begin{array}{l}28 \\
55\end{array}$ \\
\hline
\end{tabular}

Tableau 15. Identification aux acteurs de cinéma

\begin{tabular}{|c|c|c|c|c|c|c|c|c|}
\hline & \multicolumn{4}{|c|}{ Lycte Prolessionent } & \multicolumn{4}{|c|}{ Lycecegereral } \\
\hline & \multirow{3}{*}{\multicolumn{2}{|c|}{$\begin{array}{c}\text { Garçons } \\
\text { ourriers sutres } \\
23\end{array}$}} & \multirow{2}{*}{\multicolumn{2}{|c|}{$\begin{array}{c}\text { Filles } \\
\text { oavriersautres }\end{array}$}} & \multirow{2}{*}{\multicolumn{2}{|c|}{$\begin{array}{l}\text { Garpons } \\
\text { cadres autes }\end{array}$}} & \multirow{2}{*}{\multicolumn{2}{|c|}{$\begin{array}{c}\text { Files } \\
\text { cadres autres }\end{array}$}} \\
\hline & & & & & & & & \\
\hline Q. 15 n'ont pas ofacker pretitert & & & 6 & 8 & 17 & 25 & 14 & II \\
\hline $\begin{array}{l}\text { oet en acteur preflitit } \\
\text { Q. } 15 \text { racieur pretlint est }\end{array}$ & 28 & 30 & 38 & 32 & 22 & 22 & 22 & 29 \\
\hline un hoenme & 70 & 73 & 74 & 74 & 60 & 64 & 73 & 70 \\
\hline une fermene & 6 & 10 & 19 & 18 & 21 & 10 & 11 & 19 \\
\hline Q. 16 approcient chea levilelle & & & & & & & & \\
\hline $\begin{array}{l}\text { habill/physique/exprestions } \\
\text { Q.17 connaissent de luivelle }\end{array}$ & 54 & 62 & BS & 78 & 55 & 51 & 73 & 70 \\
\hline 1 element personinel su moins & 27 & 43 & 61 & 54 & 37 & 35 & 33 & 57 \\
\hline 2 elements os plus & 15 & 27 & 40 & 38 & 23 & 22 & 20 & 44 \\
\hline $\begin{array}{l}\text { Q.18 ont achele une revue pour } \\
\text { un aricke sur luivelle } \\
\text { Q.19 sont aliks voir / pournieat } \\
\text { voir }\end{array}$ & 13 & 23 & 47 & 39 & 15 & 18 & 23 & 26 \\
\hline film pev atirant avec luijelle & 53 & 57 & 75 & 68 & 55 & 50 & 57 & 64 \\
\hline
\end{tabular}

Tableau 16. Cinéma et sociabilité

\begin{tabular}{|c|c|c|c|c|c|c|c|c|}
\hline \multirow[b]{4}{*}{ jour preftert: } & \multicolumn{4}{|c|}{ Lycte Prolesstondel } & \multicolumn{4}{|c|}{ Lycte gentral } \\
\hline & \multicolumn{2}{|c|}{ Garyons } & \multirow{2}{*}{\multicolumn{2}{|c|}{$\begin{array}{c}\text { Filles } \\
\text { ouvriersautres }\end{array}$}} & \multirow{2}{*}{\multicolumn{2}{|c|}{$\begin{array}{c}\text { Garpons } \\
\text { cadres autres }\end{array}$}} & \multirow{2}{*}{\multicolumn{2}{|c|}{$\begin{array}{c}\text { Filles } \\
\text { cadres sutres }\end{array}$}} \\
\hline & ouvriers & autres & & & & & & \\
\hline & 46 & 46 & 42 & 40 & 35 & 36 & 44 & 38 \\
\hline $\begin{array}{l}\text { luadi mardi jeodi readiredi } \\
\text { nce prefferte: }\end{array}$ & $\begin{array}{l}20 \\
84\end{array}$ & $\begin{array}{l}23 \\
74\end{array}$ & 72 & ${ }_{\pi}^{23}$ & 39 & ${ }_{70}^{32}$ & $\begin{array}{l}43 \\
67\end{array}$ & $\begin{array}{l}34 \\
75\end{array}$ \\
\hline $16 \infty 19$ & 17 & 26 & 20 & 23 & 26 & 30 & 32 & 22 \\
\hline compagnie prettrte: & & & & & & & & \\
\hline $\begin{array}{l}\text { unvane ami } \\
\text { pussieun amic }\end{array}$ & 21 & 29 & 28 & 33 & 30 & 30 & 34 & 40 \\
\hline 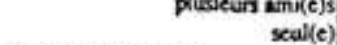 & $\begin{array}{c}65 \\
6\end{array}$ & $\begin{array}{l}66 \\
3\end{array}$ & $\begin{array}{l}65 \\
3\end{array}$ & $\begin{array}{c}56 \\
5\end{array}$ & $\begin{array}{l}52 \\
13\end{array}$ & $\frac{62}{3}$ & $\begin{array}{c}57 \\
6\end{array}$ & $\begin{array}{c}31 \\
6\end{array}$ \\
\hline $\begin{array}{l}\text { - sortie apris le film } \\
\text { - crouvens que rives at bruits }\end{array}$ & 78 & 83 & 80 & 82 & 75 & 73 & 76 & 74 \\
\hline $\begin{array}{l}\text { Strangent. } \\
\text { - Evilent films provogiant }\end{array}$ & 22 & 28 & 26 & 39 & 45 & 40 & 34 & 39 \\
\hline ces rtactions ..... & 4 & 13 & 7 & 9 & 10 & II & 9 & 16 \\
\hline
\end{tabular}


Tableau 17. Intérêt pour l'actualité cinématographique

\begin{tabular}{|c|c|c|c|c|c|c|c|c|}
\hline \multirow{4}{*}{$\begin{array}{l}\text { Les } 3 \text { derniers films vus ea salle } \\
\text { sont sorus en } 1988 \text { ou en } 1989 \\
\text { - } 2 \text { ou } 3 \text { sortis en } 88 \text { ou } 89 \\
\text { - I film au moins sorti en } 89\end{array}$} & \multicolumn{4}{|c|}{ Lycte Prolessionnel } & \multicolumn{4}{|c|}{ Lycte gentral } \\
\hline & \multicolumn{2}{|c|}{\begin{tabular}{|c|} 
Gargons \\
ouvriers autres \\
\end{tabular}} & \multicolumn{2}{|c|}{$\begin{array}{c}\text { Filles } \\
\text { ouvriersautres } \\
\end{array}$} & \multicolumn{2}{|c|}{$\begin{array}{c}\text { Garyons } \\
\text { cadres autes } \\
\end{array}$} & \multicolumn{2}{|c|}{$\begin{array}{c}\text { Fulles } \\
\text { cadres astres }\end{array}$} \\
\hline & 14 & st & 42 & 59 & 59 & 59 & 50 & 66 \\
\hline & $\begin{array}{l}72 \\
56\end{array}$ & $\begin{array}{l}75 \\
47\end{array}$ & $\begin{array}{l}68 \\
64\end{array}$ & $\begin{array}{l}80 \\
52\end{array}$ & $\begin{array}{l}84 \\
63\end{array}$ & $\begin{array}{l}80 \\
54\end{array}$ & $\begin{array}{l}76 \\
60\end{array}$ & $\begin{array}{l}74 \\
55\end{array}$ \\
\hline $\begin{array}{l}2 \text { ou } 3 \text { films classets dans les dix } \\
\text { preiniers da Box-Ortice } \\
\text { - irait voir un film primet dans un }\end{array}$ & 57 & 48 & 47 & 52 & so & 57 & 54 & 58 \\
\hline festival & 44 & 44 & 47 & 44 & 52 & 56 & 45 & 45 \\
\hline $\begin{array}{l}\text { - citent plus de } 2 \text { festivals } \\
\text { - la publicite incierait a voir }\end{array}$ & 11 & 27 & 12 & 22 & $\$ 4$ & 48 & 35 & 33 \\
\hline en flen & 60 & 53 & 56 & 56 & 58 & 52 & 52 & 50 \\
\hline
\end{tabular}

L'efficacité de l'école, telle qu'elle résulte d'une comparaison systématique entre les lycéens et celles des élèves de LEP, va bien dans le sens que l'on pouvait escompter : la qualité de la scolarisation se traduit par l'apparition de rapports « lettrés » au cinéma : le film est plus souvent rapporté à son auteur, alors que les élèves de LEP qui ont vu et apprécié Trois hommes et un couffin ignorent Colline Serreau; on apprécie le respect de la version originale de l'œuvre, fût-ce au détriment de la compréhension de l'intrigue ; on connaît davantage d'œuvres cinématographiques du passé et de réalisateurs prestigieux ; on interpose entre le regard et le film une littérature et une information critique spécialisée ; on associe plus souvent à la vision d'un film le sentiment de sérieux qui joint l'agréable au culturel. Telles sont bien les tendances. Encore faut-il noter que ce constat devrait être écrit au négatif : les lycéens sont un peu moins nombreux à ignorer La dolce vita ou Quai des brumes; ils ignorent moins fréquemment les metteurs en scène connus; et ainsi de suite. Bref, à la différence du domaine littéraire, où l'école parvient réellement, de gré ou de force, à produire des habitudes lettrées, on est loin d'assister à un transfert massif d'apprentissage dans l'univers du cinéma. L'action de l'école tend bien à induire des habitus : elle est loin d'y parvenir totalement. La nature propre du champ étudié, le cinéma, se prête peut-être plus difficilement à ses transferts. La relation au cinéma s'effectue pour tous les jeunes par deux moyens distincts: le premier, massif, est la télévision, puisque pour un film vu en salle, les jeunes interrogés en ont vu six ou sept à la télévision ; le second, exceptionnel, est la sortie au cinéma. La vision quotidienne du film à la télévision, média scolairement dévalué, voire décrié, se prête mal à la contemplation esthétisante, surtout si l'on pense qu'elle s'effectue le plus souvent dans le cadre familial, lieu géométrique des équilibres des goûts au minimum ; la sortie au cinéma, parce qu'elle est exceptionnelle, devient une occasion de se retrouver, un ingrédient dans la sociabilité des jours fériés: elle retrouve tout naturellement les chemins du cinéma offert à l'ensemble de la génération, celui dont la presse parle, celui qui passe dans les salles les mieux équipées, c'est-à-dire le chemin de l'actualité cinématographique. Entre la routine des soirées familiales et les plaisirs de la mode et de la modernité, la légitimité culturelle a du mal à frayer son chemin.

On ne voudrait pas abandonner cette enquête, sans faire observer à nos lecteurs un des plus beaux résultats obtenus dans notre carrière de professeurs d'enquête : les filles et les garçons y manifestent leurs différences d'attitude, par un choix apparemment identique, puisque les unes et les autres préfèrent des acteurs masculins... Fragilité des habitus, force des modèles de sexe.

Faut-il conclure? Ce sera, avec modestie, en attendant les résultats d'une prochaine enquête. Dans la définition de la jeunesse, la scolarisation joue un rôle très important qui 
va bien au-delà de l'imposition des connaissances et habitudes définies par les curricula formels ou réels. Mais il ne s'agit pas d'une efficacité sans limites : la famille, les médias, le groupe des pairs peuvent proposer des modèles concurrents, et le résultat de la concurrence dépend de l'univers culturel considéré. La production d'habitus homogènes dans toutes les sphères de la vie est un rêve de professeur. Aux transpositions culturelles souhaitées ou programmées, s'opposent bien des résistances : intérêts sociaux mobilisés dans des directions opposées, publics indifférents, matériaux culturels rebelles, sources de légitimité concurrentes. D'un côté les intentions des convicts scolaires, de l'autre, la vie sociale au grand air.

\section{BIBLIOGRAPHIE}

AmARA, F. - Les jeunes et la fête, Université de Provence, 1984, mémoire de maîtrise, multigr.

Baudelot, C. \& Establet, R. - Le niveau monte, Paris, Seuil, 1989 (« L'épreuve des faits »).

BOURDIEU, P. \& PASSERON, J.-C. - La reproduction. Éléments pour une théorie du système d'enseignement, Paris, Minuit, 1970 (« Le sens commun »).

DuRKheim, É. - L'évolution pédagogique en France (1938), Paris, Presses universitaires de France, $1969,2^{\mathrm{e}}$ éd. («Bibliothèque de philosophie contemporaine »)

Establet, R. - L'école est-elle rentable ?, Paris, Puf, 1987 (« Pédagogie d'aujourd'hui »).

SINGLY, F. de - Lire à 12 ans. Une enquête sur les lectures des adolescents, Paris, Nathan, 1989. 